\title{
DESARROLLO Y COSTO DE PRODUCCIÓN DE Pinus ponderosa Dougl. (ex Laws) DURANTE LAS ETAPAS DE VIVERO Y SECANO EN LA PATAGONIA ARGENTINA EFECTO DE LA SEMILLA, EL SUSTRATO Y EL CONTENEDOR
}

\author{
Sbrancia, R. ${ }^{1}$; Dezzotti, A. ${ }^{\text {; }}$ Mortoro, A. ${ }^{1}$; \\ Attis Beltrán, $H^{1,2}$ y Velásquez, A. $^{1}$
}

\section{RESUMEN}

La producción de plantas en contenedor y con sustrato fertilizado, se lleva a cabo a mayor velocidad y calidad que a raíz desnuda, pero el costo económico y ambiental es mayor. El objetivo de este trabajo fue evaluar sustratos fertilizados, de menor valor económico, y contenedores de diferentes tamaños para la producción de plantas de pino ponderosa destinadas a la forestación en la Patagonia semiárida de la Argentina.

El ensayo de desarrollo inicial se llevó a cabo en invernadero y el de establecimiento en un sitio ubicado a los $42^{\circ} 21^{\prime}$ LS y $71^{\circ} 5^{\prime}$ LO (Chubut, Argentina).

Mediante un diseño factorial se evaluó durante 2 años el diámetro, la altura, el peso, la supervivencia, la calidad y el costo de producción de las plantas, teniendo en cuenta i) semillas provenientes de conos amarillo y violeta, ii) sustratos a) turba perlita $+\mathrm{CaO}\left(5 \mathrm{~kg} / \mathrm{m}^{3}\right.$ de sustrato $)+$ fertilizante $\left(5 \mathrm{~kg} / \mathrm{m}^{3}\right.$ de sustrato), b) aserrín de pino + CaO $\left(5 \mathrm{~kg} / \mathrm{m}^{3}\right)+$ fertilizante $\left(10 \mathrm{~kg} / \mathrm{m}^{3}\right)$ y c) suelo forestal (horizonte $\mathrm{O}$ de suelo de plantación) + fertilizante $\left(10 \mathrm{~kg} / \mathrm{m}^{3}\right)$, y iii) contenedores de 120,160 y $250 \mathrm{~cm}^{3}$.

Las semillas originadas de diferentes conos no afectaron el tamaño, el peso ni los índices de calidad de las plantas. El desempeño de las plantas exhibió una interacción significativa entre el tamaño del contenedor y el tipo de sustrato. El contenedor mediano con suelo forestal fue la opción más adecuada entre las ensayadas, para desarrollar pino ponderosa desde perspectivas productiva, ecológica y económica.

Palabras clave: Pinus ponderosa, Patagonia argentina, crecimiento, índices de calidad, forestación.

\footnotetext{
1 Universidad Nacional del Comahue, Sede San Martín de los Andes, Argentina.

${ }^{2}$ Consejo Nacional de Investigaciones Científicas y Técnicas, Argentina. dezzotti@infovia.com.ar.
} 


\section{SUMMARY}

The seedlings production based on the use of container and fertilized substrate is carried out at higher speed and quality compared to bare root methods, but economic and environmental costs can be higher. Substrates composed of materials discarded from the forest activity, fertilizer and different container sizes were evaluated as alternatives for the production of Pinus ponderosa (Pinaceae) seedlings for dry land afforestation of the Patagonian steppe in Argentina.

The initial development and the establishment trials were carried out, respectively, in a greenhouse and in a farm located at $42^{\circ} 21^{\prime} \mathrm{SL}$ and $71^{\circ} 5^{\prime} \mathrm{WL}$ (Chubut, Argentina).

Through an experimental factorial design, the performance and cost of production of plants were evaluated over two years, taking into account i) seeds from yellow and violet cones, ii) the substrates a) peat perlite $+\mathrm{CaO}$ (with $5 \mathrm{~kg} / \mathrm{m}^{3}$ of substrate) + controlled release granular fertilizer $\left(5 \mathrm{~kg} / \mathrm{m}^{3}\right.$ of substrate), b) pine sawdust $+\mathrm{CaO}\left(5 \mathrm{~kg} / \mathrm{m}^{3}\right)+$ fertilizer $\left(10 \mathrm{~kg} / \mathrm{m}^{3}\right)$, and c) forest soil (O-horizon of plantation) + and fertilizer $\left(10 \mathrm{~kg} / \mathrm{m}^{3}\right)$, and iii) containers of 120,160 and $250 \mathrm{~cm}^{3}$.

Seeds originated from different cones did not affect the size, weight or quality indices of ponderosa pine seedlings. The plants performance exhibited a significant interaction between container size and substrate type. The medium-size container with fertilized plantation soil, represented the better tested option to grow Ponderosa Pine from the productive, ecological and economic points o view.

Key words: Pinus ponderosa, Argentinean Patagonia, plant growth, quality indices, afforestation. 


\section{INTRODUCCIÓN}

La plantación de árboles es una manera eficiente de desarrollar o restaurar un ecosistema forestal (Gonda et al., 2015). La plánta producida en contenedor mejora el establecimiento y extiende la temporada de plantación, particularmente en los sitios forestales subóptimos (Landis y Morgan, 2009; Hartmann et al., 2010). El desempeño de una planta durante las etapas de vivero y secano depende de factores que interactúan entre sí, que están vinculados a la semilla, el sustrato, los nutrientes, la humedad, el contenedor y el sitio forestal (Landis y Morgan, 2009). La determinación del valor de las variables que promueven diferencias de desempeño inicial de la planta es clave, porque estas diferencias se pueden mantener hasta la cosecha (Landis and Dumroese, 2006).

El contenedor con más espacio de enraizamiento determina un mayor volumen y una estructura más adecuada de la raíz, lo que brinda ventajas morfológicas y fisiológicas a la planta luego de ser plantada en el campo (South et al., 2005; Landis y Morgan, 2009). Sin embargo, la mayor cavidad del contenedor, por un lado implica más tiempo para que la raíz la explore y forme el cepellón y, por otro lado, aumenta el costo individual vinculado al mayor valor del contenedor, el medio de cultivo, el fertilizante y la irrigación, y a la menor capacidad de producción por unidad de área (Landis y Morgan, 2009). El aumento del tiempo y el costo de producción solo se justifica en sitios con alta productividad forestal (Pinto et al., 2011).

En la Patagonia noroccidental de la Argentina, las plantaciones forestales de secano se localizan sobre los faldeos extra-andinos semiáridos de las provincias de Neuquén, Río Negro y Chubut, y ocupan alrededor de 100.000 ha (MAGyP, 2014). El pino ponderosa (Pinus ponderosa (Dougl.) Laws, Pinaceae) representa el $95 \%$ de esta área, debido a su capacidad de desarrollo en las condiciones de frío y sequía que prevalecen en esta región (MAGyP, 2014).

Las plantas se producen en contenedores de 110 a $250 \mathrm{~cm}^{3}$ con fertilizante granulado de liberación controlada (FGLC). El sustrato inorgánico comúnmente utilizado es perlita y el orgánico turba (Gallo et al., 2005; Olivo y Buduba, 2006; MAGyP, 2014; Gonda et al., 2015). Estos sustratos son costosos y en particular la turba proviene de la explotación extractiva de humedales dominados por el musgo Sphagnum L. (Sphagnaceae) (Larcher and Scariot, 2009).

En el huerto semillero clonal del Instituto Nacional de Tecnología Agropecuaria (INTA, Chubut), los ramets de pino ponderosa con conos violeta maduran alrededor de 15 días antes que los ramets con conos amarillo, y en consecuencia los clones de cada fenotipo se procesan en forma separada (Gallo et al., 2005).

Los clones asociados al color del cono no difieren genotípicamente y no existe información sobre su relación con el desarrollo de esta especie (V. Mondino ${ }^{2}$, com. pers.). Sin embargo, a lo largo de la distribución geográfica natural existen ecotipos de pino ponderosa asociados al color de cono (Callaham, 2013).

\section{OBJETIVOS}

El objetivo general de este estudio fue determinar si el material que proviene del aserrado de la madera y el suelo forestal con acícula de pino, constituye un sustrato biológica y económicamente eficiente para la producción de plantas destinadas a la forestación de secano de la estepa patagónica.

La hipótesis plantea que estos sustratos son adecuados, pero la interacción con el tamaño del contenedor y el color del cono que provee la semilla afectan el desempeño de las plantas.

${ }^{2}$ Grupo de Genética Ecológica y Mejoramiento Forestal. INTA, San Carlos de Bariloche, Argentina. 


\section{MATERIAL Y MÉTODO}

\section{Área de Estudio}

El ensayo de desarrollo inicial, desde la germinación de la semilla hasta la inducción de la planta a la dormancia, se llevó a cabo en un invernadero ubicado a los $40^{\circ} 09^{\prime}$ LS y $71^{\circ} 22^{\prime}$ LO (Neuquén). El ensayo de establecimiento se desarrolló en un sitio de plantación localizado a los $42^{\circ} 21^{\prime}$ LS y $71^{\circ} 5^{\prime}$ LO (Chubut). Este sitio presenta un clima templado-frío continental, seco y ventoso, con inviernos fríos y veranos calurosos (Paruelo et al., 1998). Los valores medios de temperatura, precipitación y velocidad del viento son $8,9{ }^{\circ} \mathrm{C}, 645 \mathrm{~mm} / \mathrm{año}$ y $11 \mathrm{~km} / \mathrm{h}$, respectivamente (Figura $\mathrm{N}^{\circ} 1$ ).

El área de estudio pertenece al Distrito Occidental de la Provincia Patagónica, y está ocupada por una estepa arbustiva graminosa (Oyarzabal et al., 2018). Los suelos tienen textura gruesa, son pobres en materia orgánica, de color pardo claro o grisáceo y de reacción neutra o ligeramente alcalina, y están dominados por cantos rodados, arenas y cenizas volcánicas (Varela, 2014).

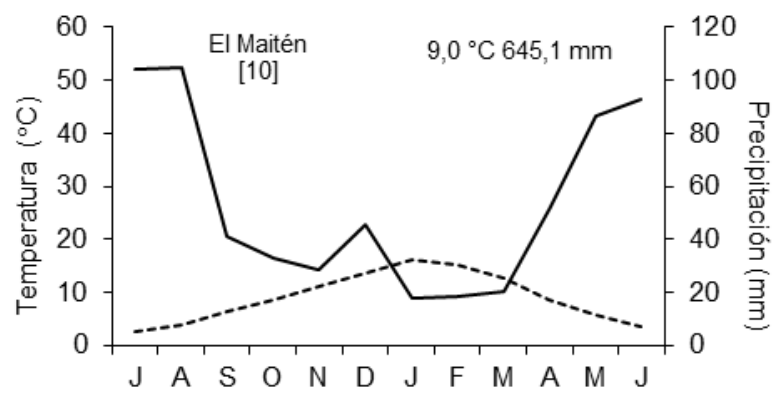

(Fuente: INTA, 2017)

Figura $N^{\circ} 1$

CLIMATOGRAMA DE LA VARIACIÓN MENSUAL DE LA TEMPERATURA (LÍNEA RAYADA) Y

PRECIPITACIÓN (LÍNEA CONTINUA) EN EL MAITÉN (42 $3^{\prime}$ LS Y 71 10' LO, 714 msnm)

PERIODO 2006 - 2015

\section{Ensayo de Desarrollo Inicial}

Los sustratos fertilizados que se evaluaron fueron:

Turba perlita (TP): Turba + perlita (proporción 3:1) + CaO $\left(5 \mathrm{~kg} / \mathrm{m}^{3}\right.$ de sustrato) + FGLC ${ }^{3}$ ( $5 \mathrm{~kg} / \mathrm{m}^{3}$ de sustrato).

Aserrín (AS): Aserrín de pino + $\mathrm{CaO}\left(5 \mathrm{~kg} / \mathrm{m}^{3}\right.$ de sustrato $)+\mathrm{FGLC}\left(10 \mathrm{~kg} / \mathrm{m}^{3}\right.$ de sustrato)

Suelo forestal (SF): Ceniza y arena volcánica + horizonte O de suelo de plantación $(1: 4)+$ FGLC (10 kg/m³ de sustrato).

El CaO se utilizó para disminuir la acidez de TP y AS. La cantidad de fertilizante de cada tratamiento varió en función del sustrato y de los aspectos consuetudinarios: TP contuvo la cantidad que comúnmente se utiliza en la región (Gonda et al., 2015) y AS y SF presentaron una mayor cantidad porque la fertilidad intrínseca y el costo son menores. Ambos aspectos justificaron la asignación de mayor cantidad de fertilizante (Landis y Morgan, 2009).

3 Basacote plus® $6 \mathrm{M}(16-8-12+2)$. 
Los valores iniciales de $\mathrm{pH}$ de los sustratos no implicaron una limitante de la fertilidad, aunque la corrección con $\mathrm{CaO}$ causó valores recomendados entre 5,5 y 6,5 (Landis y Morgan, 2009). Para cada sustrato sin $\mathrm{CaO}$ ni fertilizante, se determinó la cantidad total de $\mathrm{C}$ (método de combustión seca), $\mathrm{N}$ (método semi-micro Kjeldahl) y materia orgánica (método de calcinación), el $\mathrm{pH}$ (método potenciométrico) y la $\mathrm{CIC}$ (método de saturación con $\mathrm{NaHCO}_{3}$ y espectrometría de emisión por plasma inducido) (Jones, 2001). Luego se determinó la densidad del sustrato a partir de tres muestras de volumen conocido de cada tipo y contenedor, las cuales se pesaron en el laboratorio (balanza de precisión $0,001 \mathrm{~g}$ ), se secaron durante $24 \mathrm{~h}$ a $105^{\circ} \mathrm{C}$ (estufa) y se pesaron nuevamente (SAMLA, 1996).

Los tamaños de contenedor plástico fueron:

Grande: Bandeja de $33 \mathrm{~cm}$ de largo, $23 \mathrm{~cm}$ de ancho, $20 \mathrm{~cm}$ de profundidad, con 24 tubetes de $250 \mathrm{~cm}^{3}$ cada uno, de $5 \mathrm{~cm}$ de diámetro superior y capacidad de 325 plantas $/ \mathrm{m}^{2}$.

Mediano: Bandeja de $32 \times 27$ x 15,5 cm, 30 tubetes de $160 \mathrm{~cm}^{3}, 4,5 \mathrm{~cm}$ de diámetro, 347 plantas $/ \mathrm{m}^{2}$.

Pequeño: Bandeja de $34,5 \times 25 \times 10 \mathrm{~cm}, 35$ tubetes de $120 \mathrm{~cm}^{3}, 4,5 \mathrm{~cm}$ de diámetro, 405 plantas $/ \mathrm{m}^{2}$.

Además se evaluaron semillas de plantas con conos amarillo (AC) y violeta (VC), provenientes del huerto semillero clonal de INTA (43 $05^{\circ} \mathrm{LS}$ y $\left.71^{\circ} 28^{\prime} \mathrm{LO}\right)$. Las semillas se estratificaron a $5{ }^{\circ} \mathrm{C}$ durante 50 días, en bolsas de polietileno con arena húmeda y fungicida de contacto $\left(\mathrm{C}_{9} \mathrm{H}_{8} \mathrm{Cl}_{3} \mathrm{NO}_{2} \mathrm{~S}\right.$, dosis de $\left.1 \mathrm{~g} / \mathrm{L}\right)$.

En septiembre las semillas se sembraron en contenedores colocados en el invernadero. Las plantas emergentes se mantuvieron durante 9 meses a $26{ }^{\circ} \mathrm{C}$ fueron y regadas periódicamente. Durante los últimos 30 días las plantas se rustificaron mediante la suspensión del riego y la apertura de las cortinas del invernadero. Al finalizar esta etapa se midió el diámetro de cuello $\left(D_{c}, m m\right)$ (calibre) y la altura total $\left(H_{t}, c m\right)$ (cinta métrica) de las plantas. El peso aéreo $\left(P_{a}\right.$, $\mathrm{mg}$ ) y radicular $\left(\mathrm{P}_{\mathrm{r}}, \mathrm{mg}\right)$ se estimó a través de un método destructivo pesando nueve plantas por tratamiento, previamente secadas a $70^{\circ} \mathrm{C}$ durante $72 \mathrm{~h}$ (balanza, estufa).

Posteriormente se evaluó la calidad de las plantas a través del cálculo de:

Índice de esbeltez. $\mathrm{I}_{\mathrm{e}}=\mathrm{D}_{\mathrm{c}}\left(\left(\mathrm{H}_{\mathrm{t}} 10^{-1}\right)+2\right)^{-1}($ Schmidt-Vogt, 1981)

Índice tallo-raíz $\quad \mathrm{I}_{\mathrm{tr}}=\mathrm{P}_{\mathrm{a}} \mathrm{P}_{\mathrm{r}}^{-1}$ (Iverson, 1984)

Índice de calidad $\quad \mathrm{I}_{\mathrm{c}}=\left(\mathrm{P}_{\mathrm{a}}+\mathrm{P}_{\mathrm{r}}\right)\left(\left(\mathrm{H}_{\mathrm{t}} \mathrm{D}_{\mathrm{c}}^{-1}\right)+\left(\mathrm{P}_{\mathrm{a}} \mathrm{P}_{\mathrm{r}}^{-1}\right)\right)^{-1}$ (Dickson et al., 1960).

El diseño experimental fue factorial y completamente aleatorizado de efecto fijo con interacciones, del tipo:

$\mathrm{N}=\mathrm{n}(\mathrm{PSC})$

Donde:

$\mathrm{N}=$ Cantidad de unidades experimentales (54 contenedores con 24, 30 o 35 plantas cada uno según el tamaño del contenedor).

$\mathrm{n}=$ Cantidad de repeticiones por tratamiento (3).

$\mathrm{P}=$ Cantidad de niveles para el factor semilla (2).

$\mathrm{S}=$ Cantidad de niveles para el factor sustrato (3) 


$$
\mathrm{C}=\text { Cantidad de niveles para el factor contenedor (3). }
$$

Durante este ensayo, la humedad del sustrato se mantuvo alta y constante con un sistema automatizado de riego por micro-aspersión.

\section{Ensayo de Establecimiento}

Las plantas producidas en el ensayo de desarrollo inicial se identificaron de acuerdo a los tipos de sustrato y contenedor, y se plantaron en forma aleatoria separados entre sí $2 \mathrm{~m}$. Luego de 365 días, se estimó la supervivencia $\left(S_{\mathrm{v}}\right)$ mediante el conteo de la cantidad de plantas vivas, a las cuales se les midió $H_{t}$ y estimó el crecimiento en altura $\left(C_{a}, c m / a n ̃ o\right)$. El diseño experimental fue factorial y completamente aleatorizado de efecto fijo con interacciones del tipo:

$$
\mathrm{N}=\mathrm{n}(\mathrm{SC})
$$

Donde:

$\mathrm{N}=$ Cantidad de unidades experimentales (27 contenedores de 24, 30 o 35 plantas según el tamaño del contenedor).

$\mathrm{n}=$ Cantidad de repeticiones por tratamiento (3).

$\mathrm{S}=$ Cantidad de niveles para el factor sustrato (3).

$\mathrm{C}=$ Cantidad de niveles para el factor contenedor (3).

En ambos ensayos los datos se analizaron a través de pruebas paramétricas porque cumplieron con los supuestos del ANOVA. El efecto sobre el desempeño de las plantas de los diferentes factores, niveles y las interacciones se analizó mediante modelos desbalanceados (ensayo de desarrollo inicial) y balanceados (ensayo de establecimiento), a través de ANOVA multifactorial y pruebas de comparaciones múltiples.

\section{Costo de Producción}

El costo de producción de la planta de cada tratamiento se obtuvo conociendo en la región y en un momento dado, el valor de mercado del sustrato, el fertilizante, el contenedor y el transporte, el volumen de sustrato y la capacidad de plantas por contenedor. En los casos del SF y AS, el valor solo incluyó el de la carga, el transporte y la descarga. El costo se comparó entre tratamientos y se expresó en términos relativos, para que el valor de la estimación no pierda vigencia debido a variaciones de los precios de los bienes y servicios.

\section{RESULTADOS Y DISCUSIÓN}

\section{Ensayo de Desarrollo Inicial}

Los sustratos, evaluados sin el agregado de $\mathrm{CaO}$ ni fertilizante, presentaron características físicas y químicas diferentes que fueron los esperados según la composición (Landis y Morgan, 2009).

TP presentó valores altos de $\mathrm{N}$, materia orgánica y CIC, intermedios de densidad y C, y bajos de $\mathrm{C}: \mathrm{N}$ y $\mathrm{pH}$.

AS presentó valores altos de $\mathrm{C}, \mathrm{C}: \mathrm{N}$, materia orgánica y $\mathrm{CIC}$, intermedios de $\mathrm{pH}$ y bajos de densidad y $\mathrm{N}$.

SF presentó valores altos de densidad y $\mathrm{pH}$, intermedios de $\mathrm{N}$ y bajos de $\mathrm{C}, \mathrm{C}: \mathrm{N}$, materia orgánica y $\mathrm{CIC}$ (Cuadro $\mathrm{N}^{\circ} 1$ ). 
La densidad de AS y SF estuvo dentro del rango de otros sustratos alternativos utilizados para producir pino ponderosa en la región (Olivo y Buduba, 2006). Aunque no se estimó la relación entre la cantidad de macro y microporos de los sustratos, la proporción relativa entre porosidad de aireación y de retención de agua fue satisfactoria dada la heterogeneidad en tamaño de sus componentes (Landis y Morgan, 2009).

\section{Cuadro $\mathrm{N}^{\circ} 1$}

DENSIDAD, CANTIDAD DE C, N Y MATERIA ORGÁNICA (MO), PH Y CIC DE LA TURBA PERLITA (TP), EL ASERRÍN (AS) Y EL SUELO FORESTAL (SF)

\begin{tabular}{|c|c|c|c|c|}
\hline \multirow{2}{*}{\multicolumn{2}{|c|}{ Variable }} & \multicolumn{3}{|c|}{ Sustrato } \\
\hline & & \multirow{2}{*}{$\begin{array}{c}\text { TP } \\
0,117 \\
0,003 \\
\text { a } \\
9\end{array}$} & \multirow{2}{*}{$\begin{array}{c}\text { AS } \\
0,088 \\
0,001 \\
\text { b } \\
9\end{array}$} & \multirow{2}{*}{$\begin{array}{c}\text { SF } \\
0,471 \\
0,009 \\
\text { c } \\
9\end{array}$} \\
\hline $\begin{array}{l}\text { Densidad } \\
\left(\mathrm{g} / \mathrm{cm}^{3}\right)\end{array}$ & $\begin{array}{c}\mathbf{X} \\
\mathrm{EE} \\
\mathrm{GH} \\
\mathrm{n} \\
\end{array}$ & & & \\
\hline \multicolumn{2}{|c|}{ C (\%) } & 31,1 & 49,7 & 8,4 \\
\hline \multicolumn{2}{|c|}{$\mathrm{N}(\%)$} & 0,84 & 0,05 & 0,44 \\
\hline \multicolumn{2}{|c|}{$\mathrm{C}: \mathrm{N}$} & 37 & 994 & 19 \\
\hline \multicolumn{2}{|c|}{$\mathrm{MO}(\%)$} & 79,8 & 83,2 & 23,0 \\
\hline \multicolumn{2}{|c|}{$\mathrm{pH}$} & 3,9 & 4,7 & 5,5 \\
\hline \multicolumn{2}{|c|}{$\mathrm{CIC}(\mathrm{cmol} / \mathrm{kg})$} & 75,9 & 78,1 & 20,8 \\
\hline
\end{tabular}

Los valores representan la medición del sustrato sin CAO ni fertilizante.

Se indica la media (X), el error estándar (EE), los grupos homogéneos (GH) y el tamaño de la muestra $(n)$.

Letras desiguales indican diferencias significativas (ANOVA; $F_{2,26}=1613,4 ; P<0,001$ )

$H_{t}$ media de las plantas fue $9,4 \mathrm{~cm}(E E=0,38 ; n=54)$. $H_{t}$ no difirió significativamente entre las semillas provenientes de AC y VC (ANOVA, $p<0,05)$.

$\mathrm{H}_{\mathrm{t}}$ fue 79,6 y $38,6 \%$ significativamente mayor en SF y TP, respectivamente, que en AS (ANOVA, $p<0,05$ ).

$H_{t}$ fue 25,0 y $41,5 \%$ mayor en el contenedor mediano (M) y grande $(G)$, respectivamente, que en el pequeño $(P)(A N O V A, p<0,05)$ (Cuadro $N^{\circ} 2$, Figura $\left.N^{\circ} 2\right)$.

$\mathrm{H}_{\mathrm{t}}$ exhibió una interacción significativa entre la del contenedor y el sustrato (ANOVA, $\mathrm{p}<$ 0,05 ).

En AS, $H_{t}$ fue entre 15,4 y $34,0 \%$ significativamente mayor en $M$ y $G$ que en $P$.

En SF, $\mathrm{H}_{\mathrm{t}}$ fue 31,8 y 47,1 \% significativamente mayor en $\mathrm{M}$ y $\mathrm{G}$, respectivamente, que $\mathrm{P}$.

En TP, $H_{t}$ fue 23,6 y 40,1\% significativamente mayor en $M$ y $G$, respectivamente, que en $\mathrm{P}$ (prueba de Tukey, $\mathrm{p}<0,05)\left(\right.$ Cuadro $\mathrm{N}^{\circ} 2$, Figura $\left.\mathrm{N}^{\circ} 3\right)$.

$\mathrm{H}_{\mathrm{t}}$ difirió significativamente entre los tratamientos de contenedor y sustrato (ANOVA, $\left.\mathrm{F}_{8,53}=46,7 ; \mathrm{p}<0,001 ; \mathrm{n}=6\right)$.

$H_{t}$ fue $131,1 \%$ mayor en $M$ y G con SF que en $P$ y con AS, que representó el tratamiento con menor desempeño de las plántulas (prueba de Tukey, $p<0,05$ ) (Figura $N^{\circ} 4$ ). 


\section{Cuadro $\mathrm{N}^{\circ} 2$}

ANÁLISIS DE VARIANZA DE LA ALTURA $\left(\mathrm{H}_{T}\right)$, EL DIÁMETRO $\left(\mathrm{D}_{\mathrm{C}}\right)$, EL PESO AÉREO $\left(\mathrm{P}_{\mathrm{A}}\right)$ Y RADICULAR

$\left(P_{R}\right)$, Y LOS ÍNDICES DE ESBELTEZ (IE), TALLO-RAÍZ (ITR) Y CALIDAD (IC)

DE PINO PONDEROSA DURANTE EL ENSAYO DE DESARROLLO INICIAL

\begin{tabular}{|c|c|c|c|c|c|c|c|c|c|}
\hline \multirow[b]{2}{*}{ FV } & \multirow[b]{2}{*}{ gl } & \multirow[b]{2}{*}{$\mathbf{n}$} & \multicolumn{7}{|c|}{$F(p)$} \\
\hline & & & $\begin{array}{c}\mathrm{H}_{\mathrm{t}} \\
(\mathrm{cm})\end{array}$ & $\begin{array}{c}D_{c} \\
(\mathrm{~mm})\end{array}$ & $\begin{array}{c}\mathrm{Pa} \\
(\mathrm{mg})\end{array}$ & $\begin{array}{c}P_{r} \\
(\mathrm{mg})\end{array}$ & $I_{t r}$ & $\mathrm{Ie}_{\mathrm{e}}$ & $I_{c}$ \\
\hline$P$ & 1 & 27 & $\begin{array}{c}3,6 \\
(0,067)\end{array}$ & $\begin{array}{c}0,1 \\
(0,327)\end{array}$ & $\begin{array}{c}3,8 \\
(0,061)\end{array}$ & $\begin{array}{c}0,1 \\
(0,747)\end{array}$ & $\begin{array}{c}2,8 \\
(0,102)\end{array}$ & $\begin{array}{c}0,1 \\
(0,723)\end{array}$ & $\begin{array}{c}0,1 \\
(0,878)\end{array}$ \\
\hline$S$ & 2 & 18 & $\begin{array}{c}149,4 \\
(<0,001)\end{array}$ & $\begin{array}{c}154,0 \\
(<0,001)\end{array}$ & $\begin{array}{c}69,8 \\
(<0,001)\end{array}$ & $\begin{array}{c}60,0 \\
(<0,001)\end{array}$ & $\begin{array}{c}13,0 \\
(<0,001)\end{array}$ & $\begin{array}{c}98,1 \\
(<0,001)\end{array}$ & $\begin{array}{c}82,1 \\
(<0,001)\end{array}$ \\
\hline C & 2 & 18 & $\begin{array}{c}53,6 \\
(<0,001)\end{array}$ & $\begin{array}{c}23,8 \\
(<0,001)\end{array}$ & $\begin{array}{c}21,4 \\
(<0,001)\end{array}$ & $\begin{array}{c}41,1 \\
(<0,001)\end{array}$ & $\begin{array}{c}1,7 \\
(0,200)\end{array}$ & $\begin{array}{c}7,5 \\
(0,002)\end{array}$ & $\begin{array}{c}36,0 \\
(<0,001)\end{array}$ \\
\hline PS & 2 & 9 & $\begin{array}{c}0,8 \\
(0,478)\end{array}$ & $\begin{array}{c}0,4 \\
(0,675)\end{array}$ & $\begin{array}{c}3,2 \\
(0,054)\end{array}$ & $\begin{array}{c}0,7 \\
(0,522)\end{array}$ & $\begin{array}{c}1,7 \\
(0,206)\end{array}$ & $\begin{array}{c}0,1 \\
(0,910)\end{array}$ & $\begin{array}{c}1,2 \\
(0,327)\end{array}$ \\
\hline PC & 2 & 9 & $\begin{array}{c}0,1 \\
(0,935)\end{array}$ & $\begin{array}{c}0,2 \\
(0,856)\end{array}$ & $\begin{array}{c}0,4 \\
(0,653)\end{array}$ & $\begin{array}{c}0,4 \\
(0,692)\end{array}$ & $\begin{array}{c}0,2 \\
(0,787)\end{array}$ & $\begin{array}{c}0,4 \\
(0,707)\end{array}$ & $\begin{array}{c}0,2 \\
(0,787)\end{array}$ \\
\hline SC & 4 & 6 & $\begin{array}{c}3,3 \\
(0,027)\end{array}$ & $\begin{array}{c}7,5 \\
(<0,001)\end{array}$ & $\begin{array}{c}5,8 \\
(<0,001)\end{array}$ & $\begin{array}{c}5,0 \\
(0,003)\end{array}$ & $\begin{array}{c}0,4 \\
(0,831)\end{array}$ & $\begin{array}{c}5,9 \\
(0,001)\end{array}$ & $\begin{array}{c}6,7 \\
(<0,001)\end{array}$ \\
\hline PSC & 4 & 3 & $\begin{array}{c}2,3 \\
(0,077)\end{array}$ & $\begin{array}{c}1,7 \\
(0,166)\end{array}$ & $\begin{array}{c}3,3 \\
(0,022)\end{array}$ & $\begin{array}{c}5,6 \\
(0,001)\end{array}$ & $\begin{array}{c}0,9 \\
(0,499)\end{array}$ & $\begin{array}{c}0,8 \\
(0,532)\end{array}$ & $\begin{array}{c}6,5 \\
(<0,001)\end{array}$ \\
\hline
\end{tabular}

Se indica la fuente de variación (FV: P: Semilla, S: Sustrato, C: Contenedor; PS, PC y SC: Interacciones entre P, y C), los grados de libertad ( $(\mathrm{l})$ ), el tamaño de la muestra $(n)$, el cociente $(F)$ y el nivel de probabilidad $(p)$,
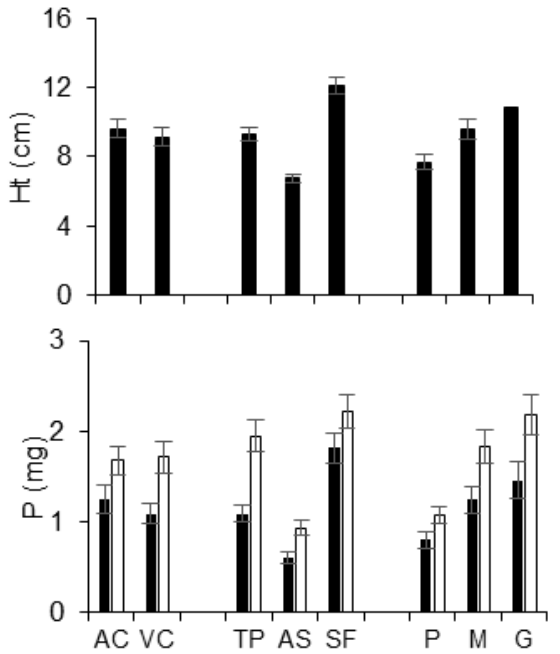
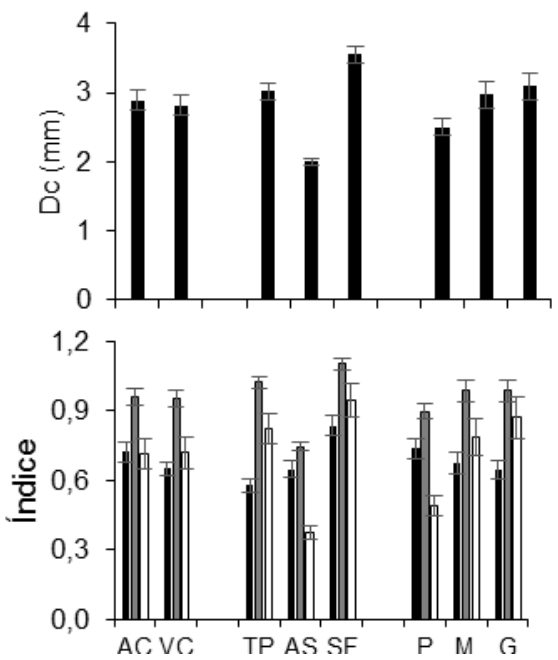

Altura $(\mathrm{Ht})$; Diámetro $(\mathrm{Dc})$; Peso aéreo (barra negra) y radicular (barra blanca) $(\mathrm{P})$, e Índices de esbeltez (barra negra), tallo-raíz (barra gris) y calidad (barra blanca), con relación a la semilla (AC: cono amarillo, VC: cono violeta), el Sustrato (TP: turba perlita, AS: aserrín, SF: suelo forestal) y el Contenedor (P: pequeño, M: mediano, G: grande).

Las barras verticales indican el error estándar y las letras diferentes las diferencias significativas entre niveles dentro del factor (Prueba de Tukey, $p<0,05 ; n=3$ ). 
La altura menor de las plantas, comparada con la obtenida con otros sustratos alternativos en la región (Olivo y Buduba, 2006), no sería desventajosa porque aumentaría la supervivencia en áreas frías y secas (MAGyP, 2014).

En estas condiciones, McTague and Tinus (1996) sugieren una altura cercana a los 16 $\mathrm{cm}$. En cambio, la altura menor afectaría el desarrollo de la planta en sitios de plantación mejores, teniendo en cuenta la correlación entre la altura inicial y la de los primeros 15 años (Buamscha et al., 2012).

Sin embargo, en la Patagonia prácticamente no se planta pino ponderosa en sitios de calidad alta (MAGyP, 2014).

En el sitio de plantación, un indicador más adecuado de la supervivencia, el crecimiento de altura y la producción de madera es $D_{c}$ (Buamscha et al., 2012).

$D_{c}$ medio de las plántulas fue 2,9 mm $(E E=0,10 ; n=54)$.

$D_{c}$ no difirió significativamente entre las semillas provenientes de los conos amarillos y violetas (ANOVA, $\mathrm{p}<0,05)$.

$D_{c}$ fue 77,7 y $50,9 \%$ significativamente mayor en SF y TP, respectivamente, que en AS (ANOVA, $p<0,05$ ).

$D_{c}$ fue $21,1 \%$ significativamente mayor en el contenedor $M$ y $G$ que en $P$ (ANOVA, $p<$ $0,05)$ (Cuadro $N^{\circ} 2$, Figura $N^{\circ} 2$ ).

$D_{c}$ presentó una interacción significativa entre el contenedor y el sustrato (ANOVA, $p<$ $0,05)$.

En $A S, D_{c}$ no difirió entre los diferentes contenedores.

En SF, $D_{c}$ fue entre 29,1 y $32,3 \%$ significativamente mayor en el contenedor $M$ y $G$ que en $\mathrm{P}$.

En TP, $D_{c}$ fue 17,4 y $34,5 \%$ significativamente mayor en el contenedor $M$ y $G$, respectivamente, que en $\mathrm{P}$ (prueba de Tukey, $\mathrm{p}<0,05)$ (Cuadro $\mathrm{N}^{\circ} 2$, Figura $\mathrm{N}^{\circ} 3$ ).

$D_{c}$ difirió significativamente entre los tratamientos de contenedor y sustrato (ANOVA, $F_{8,5}=$ $48,2 ; p<0,001 ; n=6)$.

$D_{c}$ fue $93,4 \%$ significativamente mayor en el contenedor $M$ y $G$ con SF que en el $P$ y $M$ con AS, que representaron los tratamientos con menor desempeño de las plántulas (prueba de Tukey, $\mathrm{p}<0,05)$ (Figura $\left.\mathrm{N}^{\circ} 4\right)$.

El valor de $D_{c}$ de los tratamientos con mejor desempeño superó $4 \mathrm{~mm}$, y fueron similares a los obtenidos para $P$. ponderosa con sustratos alternativos orgánico (fibra de coco y turba de Sphagnum) e inorgánico (pumita, vermiculita y perlita) y a los recomendados en la región para realizar el trasplante (Olivo y Buduba, 2006). 

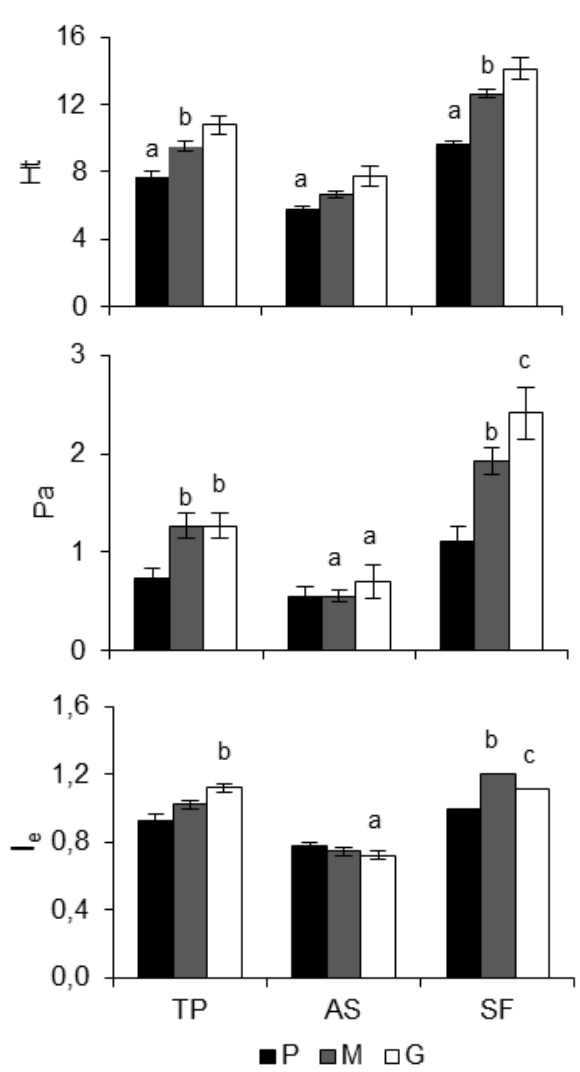
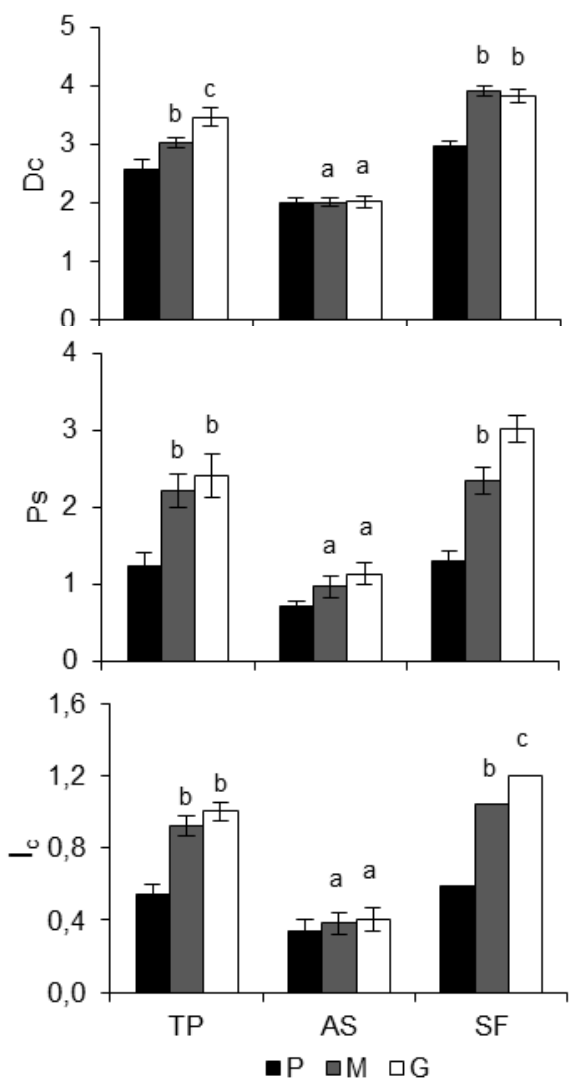

Altura $\left(H_{t}\right)$, Diámetro $\left(D_{c}\right)$, Peso Aéreo $\left(P_{a}\right)$ y Radicular $\left(P_{r}\right)$ e Índices de Esbeltez $\left(\mathrm{I}_{e}\right)$ y Calidad $\left(I_{c}\right)$ con relación al Sustrato (Tp: Turba Perlita, As: Aserrín, Sf: Suelo Forestal) y el Contenedor ( $P$ : Pequeño, M: Mediano, G: Grande).

Las barras verticales indican el error estándar y las letras diferentes las diferencias significativas entre el tamaño del contenedor dentro del tipo de sustrato (Prueba de Tukey, $\mathrm{P}<0,05 ; \mathrm{N}=3$ ).

Figura $\mathrm{N}^{\circ} 3$

RESPUESTA DE LAS PLANTAS SEGÚN LAS DIFERENTES VARIABLES EVALUADAS EN EL ENSAYO DE DESARROLLO INICIAL SEGÚN SUSTRATO Y CONTENEDOR

$P_{a}$ medio de las plantas fue $1,2 \mathrm{mg}(E E=0,09 ; n=54)$.

$\mathrm{P}_{\mathrm{a}}$ no difirió significativamente entre las semillas provenientes de los conos amarillo y violeta (ANOVA, $p<0,05)$.

$\mathrm{P}_{\mathrm{a}}$ fue 201,2 y $81,0 \%$ significativamente mayor en SF y TP, respectivamente, que en AS.

$\mathrm{P}_{\mathrm{a}}$ fue $69,4 \%$ significativamente mayor en el contenedor $\mathrm{M}$ y $\mathrm{G}$ que en $\mathrm{P}$ (ANOVA, $\mathrm{p}<$ $0,05)$ (Cuadro $\mathrm{N}^{\circ} 2$, Figura $\mathrm{N}^{\circ} 2$ ).

$\mathrm{P}_{\mathrm{a}}$ presentó una interacción significativa entre la del contenedor y el sustrato (ANOVA, $\mathrm{p}$ $<0,05)$.

En $A S, P_{a}$ no difirió significativamente entre los diferentes contenedores (prueba de Tukey, $p \geq 0,05$ ). 
En SF, $P_{a}$ fue entre 72,8 y $116,3 \%$ significativamente mayor en el contenedor $M$ y $G$ que en $\mathrm{P}$.

En TP, $P_{a}$ fue entre 70,8 y $71,4 \%$ significativamente mayor en el contenedor $M$ y $G$ que en $\mathrm{P}$ (prueba de Tukey, $\mathrm{p}<0,05)\left(\right.$ Cuadro $\mathrm{N}^{\circ} 2$, Figura $\mathrm{N}^{\circ} 3$ ).

$\mathrm{P}_{\mathrm{a}}$ difirió significativamente entre los tratamientos de contenedor y sustrato (ANOVA, $\mathrm{F}_{8,53}$ $=19,3 ; p<0,001 ; n=6)$.

$P_{a}$ fue $296,7 \%$ significativamente mayor en el contenedor $M$ y $G$ con SF que en $P$ y $M$ con AS, que representaron los tratamientos con menor desempeño de las plantas (prueba de Tukey, $\mathrm{p}<0,05)$ (Figura $\mathrm{N}^{\circ} 4$ ).

$\mathrm{P}_{\mathrm{r}}$ medio de las plántulas fue $1,7 \mathrm{mg}(\mathrm{EE}=0,11 ; \mathrm{n}=54)$.

$\mathrm{P}_{\mathrm{r}}$ no difirió significativamente entre las semillas provenientes de los conos amarillo y violeta (ANOVA, $p \geq 0,05$ ).

$P_{r}$ fue 138,1 y $109,1 \%$ significativamente mayor en SF y TP, respectivamente, que en AS (ANOVA, $\mathrm{p}<0,05$ ).

$P_{r}$ fue 90,3 y 134,4\% significativamente mayor en el contenedor M y $G$, respectivamente, que en $\mathrm{P}$ (ANOVA, $\mathrm{p}<0,05)$ (Cuadro $\mathrm{N}^{\circ} 2$, Figura $\mathrm{N}^{\circ} 2$ ).

$P_{r}$ presentó una interacción significativa entre la del contenedor y el sustrato (ANOVA, $p<$ $0,05)$.

En AS, $P_{r}$ no difirió significativamente entre los diferentes contenedores (prueba de Tukey, $p \geq 0,05$ ).

En SF, $P_{r}$ fue 79,6 y 131,3 \% significativamente mayor en el contenedor $M$ y $G$, respectivamente, que en $P$.

En TP, $P_{r}$ fue entre 77,9 y $94,7 \%$ significativamente mayor en el contenedor $M$ y $G$ que en $\mathrm{P}$ (prueba de Tukey, $\mathrm{p}<0,05)\left(\right.$ Cuadro $\mathrm{N}^{\circ} 2$, Figura $\mathrm{N}^{\circ} 3$ ).

$\mathrm{P}_{\mathrm{r}}$ difirió significativamente entre los tratamientos de contenedor y sustrato (ANOVA, $\mathrm{F}_{8,53}$ $=20,7, p<0,001, n=6)$.

$P_{r}$ fue $324,8 \%$ significativamente mayor en el contenedor $G$ y con $S F$ que en $P, M$ y $G$ con AS y $\mathrm{P}$ con TP, que representaron los tratamientos con menor desempeño de las plantas (prueba de Tukey, $\mathrm{p}<0,05)\left(\right.$ Figura $\left.\mathrm{N}^{\circ} 4\right)$.

$\mathrm{P}_{\mathrm{a}}$ y $\mathrm{P}_{\mathrm{r}}$ de los distintos tratamientos estuvieron dentro del rango de los valores obtenidos por Olivo y Buduba (2006) y Buamscha et al. (2012).

$\mathrm{P}_{\mathrm{a}}$ y $\mathrm{P}_{\mathrm{r}}$ son indicadores de la calidad de la planta cuando estas variables se relacionan entre sí, aunque en forma aislada permiten analizar el efecto del sustrato y el tubete sobre la asignación de biomasa aérea y radicular (Buamscha et al., 2012).

$I_{\mathrm{tr}}, \mathrm{I}_{\mathrm{e}}$ e $\mathrm{I}_{\mathrm{c}}$ no se diferenciaron entre las semillas provenientes de los conos amarillo y violeta (ANOVA, $p \geq 0,05$ ).

Itr no difirió entre las que se desarrollaron en los diferentes tamaños de contenedor (ANOVA, $p \geq 0,05$ ), pero si entre las plantas producidas con los distintos sustratos (ANOVA, $p<0,05)$.

$I_{e}$ y $I_{c}$ presentaron diferencias entre las plantas producidas con los distintos sustratos (ANOVA, $p<0,05$ ) y y contenedores (ANOVA, $p<0,05$ ), y existieron interacciones entre ambos factores (ANOVA, $\mathrm{p}<0,05)\left(\right.$ Cuadro $\mathrm{N}^{\circ} 2$, Figuras $\mathrm{N}^{\circ} 2,3$ y 4).

La alta proporción de raíz representada por el valor de $I_{t r}$ bajo fue indicativo de la aptitud de los individuos para la plantación en secano; Olivo y Buduba (2006) obtuvieron valores de $I_{t r}$ mayores. 

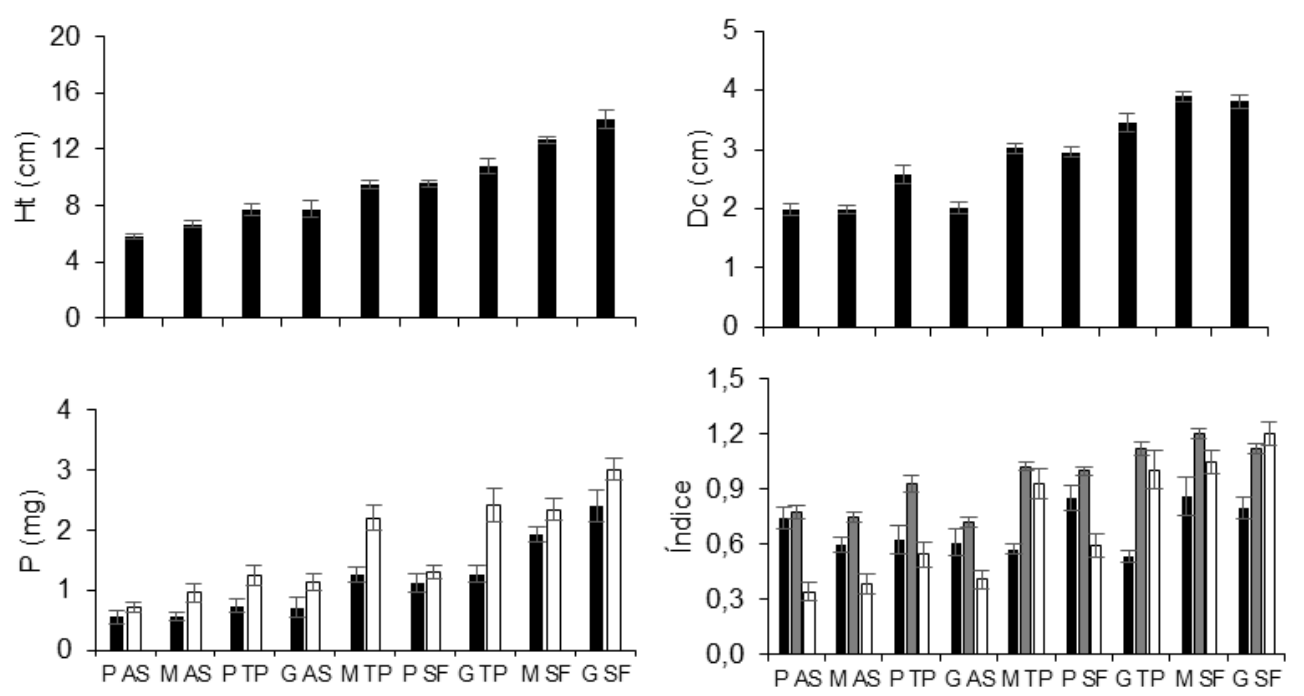

Altura $\left(H_{t}\right)$, Diámetro $\left(D_{c}\right)$, Peso $(P)$ Aéreo (Barra Negra) y Radicular (Barra Blanca) e Índices Tallo-Raíz (Barra Negra), de Esbeltez (Barra Gris) y Calidad (Barra Blanca) con relación al Tamaño del Contenedor (P: Pequeño, M: Mediano, G: Grande) y El Tipo de Sustrato (Tp: Turba Perlita, As: Aserrín, Sf: Suelo Forestal).

Las barras verticales indican el error estándar y las letras diferentes las diferencias significativas entre tratamientos (Prueba de Tukey, $\mathrm{P}<0,05 ; \mathrm{N}=3$ ).

Figura $\mathrm{N}^{\circ} 4$

\section{RESPUESTA DE LAS PLANTAS SEGÚN LAS DIFERENTES VARIABLES EVALUADAS EN EL ENSAYO DE DESARROLLO INICIAL SEGÚN SUSTRATO Y CONTENEDOR}

En plantaciones en sitios de alta calidad $\mathrm{P}_{\mathrm{a}}$ debería ser hasta el doble que $\mathrm{P}_{\mathrm{r}}$, pero en aquellos con déficit hídrico el trasplante exige un mayor desarrollo radicular (Quiroz et al., 2014). El valor de $\mathrm{l}_{\mathrm{e}}$ debe ser cercano a 1 (Buamscha et al. 2012), lo que ocurrió en todos los tratamientos a excepción de aquellos con AS que fue menor. En la región, Olivo y Buduba (2006) obtuvieron para pino ponderosa valores de $I_{e}$ similares con sustratos alternativos. Los valores altos de $I_{c}$ indicaron la mejor calidad de la planta y desempeño de ellas en la plantación (Buamscha et al., 2012). SF con M $(1,04)$ y $G(1,20)$ representaron las mejores opciones, cuyos valores superaron los obtenidos con sustratos alternativos y tubete pequeño de $120 \mathrm{~cm}^{3}(0,59-0,85)$ (Olivo y Buduba, 2006).

\section{Ensayo de Establecimiento}

Durante el ensayo de desarrollo inicial no se observaron diferencias significativas entre AC y VC en ninguna de las variables y, en consecuencia, el factor semilla no se tuvo en cuenta durante el ensayo de establecimiento. Además, una de las repeticiones de este último ensayo tuvo una supervivencia nula, que se asoció a factores externos a las condiciones naturales del ensayo, por lo tanto no se la consideró en el análisis.

$C_{a}$ medio fue $3,47 \mathrm{~cm} /$ año ( $E E=0,49 ; n=18$ ).

$\mathrm{C}_{\mathrm{a}}$ de las plantas provenientes del contenedor $\mathrm{G}$ fue $4,60 \mathrm{~cm} / \mathrm{año}$ que representó un valor $127,9 \%$ mayor al de las provenientes del contenedor $P$ (prueba de Tukey, $p<0,05$ ).

$\mathrm{C}_{\mathrm{a}}$ de las plantas provenientes de SF fue 5,17 cm/año que representó un valor 96,8 \% mayor al de las provenientes de AS y TP (prueba de Tukey, $p<0,05$ ).

$\mathrm{C}_{\mathrm{a}}$ no presentó una interacción significativa entre el contenedor y el sustrato (ANOVA, $p \geq$ 
0,05) (Cuadro $N^{\circ} 3$, Figura $N^{\circ} 5$ ).

$\mathrm{C}_{\mathrm{a}}$ difirió significativamente entre los tratamientos de contenedor y sustrato (ANOVA, $\mathrm{F}_{8,17}$ $=3,26 ; \mathrm{p}=0,049 ; \mathrm{n}=2$ ).

$\mathrm{C}_{\mathrm{a}}$ en el contenedor $\mathrm{G}$ con SF fue $6,6 \mathrm{~cm}^{2}$ año $^{-1}$, que representó un valor en $417,8 \%$ significativamente mayor que $\mathrm{P}$ con TP (prueba de Tukey, $\mathrm{p}<0,05$ ). En el resto de los tratamientos $C_{a}$ varió entre 2,0 (P y AS) y 6,2 (M y SF) (Figura $N^{\circ} 6$ ).

$S_{v}$ media fue $77,3 \%(E E=2,48 ; n=18)$.

$S_{v}$ no difirió significativamente entre las plantas que provinieron de los contenedores $P, M$ y $G$ (ANOVA, $p \geq 0,05$ ).

$S_{v}$ de las plantas no difirió significativamente entre las que provinieron de la SF, TP y AS (ANOVA, $p \geq 0,05$ ).

$S_{v}$ no presentó una interacción significativa entre el contenedor y el sustrato (ANOVA, $p \geq$ $0,05)$ (Cuadro $N^{\circ} 3$, Figura $N^{\circ} 5$ ).

$\mathrm{S}_{\mathrm{v}}$ no difirió significativamente entre los tratamientos de contenedor y sustrato (ANOVA, $\mathrm{F}_{8,17}=0,59 ; \mathrm{p}=0,765 ; \mathrm{n}=2$ ) (Figura $\mathrm{N}^{\circ}$ 6).

En el sitio de plantación, al igual que en el vivero, las plantas con SF presentaron los valores mayores de tamaño, peso y crecimiento, y en general, de los índices de desempeño. Estudios previos mostraron resultados satisfactorios combinando materiales más económicos y tradicionales (Gerding et al., 1996; Olivo y Buduba, 2006; Bárbaro et al., 2014).

En ambos ensayos, las plantas de los contenedores de mayor volumen y profundidad presentaron el mejor desempeño. Resultados equivalentes fueron señalados en la producción de Pinus L. (South et al., 2005; Domínguez-Lerena et al., 2006; Bernaola-Paucar et al., 2015) y otras especies (Close et al., 2010; Dobner et al., 2013).

\section{Costo de Producción}

AS - P fue el tratamiento que presentó el menor costo de producción por unidad de planta y TP - G exhibió el mayor costo de producción, con un valor relativo $172 \%$ mayor al primero (Cuadro $\mathrm{N}^{\circ} 4$ ).

En AS, el contenedor M y G representaron un valor por planta 33,2 y 84,1\% mayor al del pequeño, respectivamente, mientras que el grande representó un valor 38,2\% mayor al de $M$.

En SF, los contenedores M y G representaron un valor por planta 33,4 y $85,0 \%$ mayor al de $\mathrm{P}$, respectivamente, mientras que $\mathrm{G}$ representó un valor $38,7 \%$ mayor al de $\mathrm{M}$.

En TP, los contenedores M y G representaron un valor por planta 34,3 y 89,6\% mayor al de $P$, respectivamente, mientras que $G$ representó un valor $41,2 \%$ mayor al de $M$.

En P, SF y TP representaron un valor por planta 4,9 y $43,5 \%$ mayor al de AS, respectivamente, mientras que TP representó un valor 36,8 \% mayor al de SF.

En el contenedor M, SF y TP representaron un valor por planta 5,0 y 44,7 \% mayor a AS, respectivamente, mientras que TP representó un valor $37,8 \%$ mayor con respecto de SF.

En el contenedor G, SF y TP representaron un valor por planta 5,3 y 47,8 \% mayor al de AS, respectivamente, mientras que TP representó un valor $40,3 \%$ mayor con respecto al de SF. 
Cuadro $\mathrm{N}^{\circ} 3$

ANÁLISIS DE VARIANZA MULTIFACTORIAL DEL CRECIMIENTO EN ALTURA $\left(\mathrm{C}_{A}\right)$

$Y$ LA SOBREVIVENCIA (Sv) DURANTE EL ENSAYO DE ESTABLECIMIENTO

\begin{tabular}{|c|c|c|c|c|}
\hline \multirow{2}{*}{ FV } & \multirow{2}{*}{$\mathbf{g l}$} & $\mathbf{n}$ & \multicolumn{2}{|c|}{$\mathbf{F}(\mathbf{p})$} \\
\cline { 4 - 5 } & & \multirow{2}{*}{$\begin{array}{c}\mathbf{C}_{\mathbf{a}} \\
\text { (cm/año) }\end{array}$} & $\begin{array}{c}\mathbf{S}_{\mathbf{v}} \\
(\%)\end{array}$ \\
\hline $\mathrm{S}$ & 2 & 6 & $\begin{array}{c}6,21 \\
(0,020)\end{array}$ & $\begin{array}{c}0,64 \\
(0,551)\end{array}$ \\
\hline $\mathrm{C}$ & 2 & 6 & $\begin{array}{c}5,03 \\
(0,034)\end{array}$ & $\begin{array}{c}1,37 \\
(0,302)\end{array}$ \\
\hline SC & 4 & 2 & $\begin{array}{c}0,90 \\
(0,503)\end{array}$ & $\begin{array}{c}0,18 \\
(0,946)\end{array}$ \\
\hline
\end{tabular}

Se indica la fuente de variación (FV) (S: Sustrato, C: Contenedor), los grados de libertad (GL), el tamaño de la muestra ( $n$ ), el cociente $F$ y el nivel de probabilidad ( $p$ )
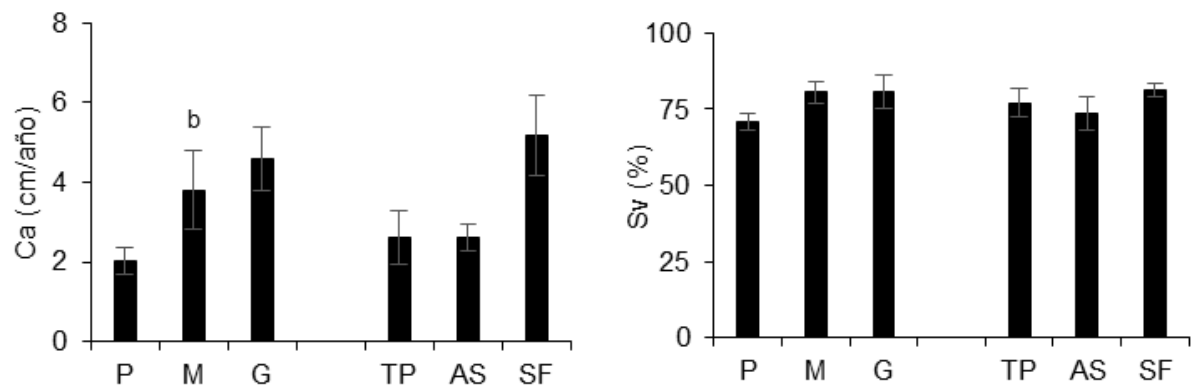

Crecimiento en altura $\left(\mathrm{C}_{\mathrm{A}}\right)$ y sobrevivencia $(\mathrm{S} v)$ con relación al tamaño del contenedor (P: Pequeño, M: Mediano, G: Grande) y el tipo de sustrato (TP: Turba perlita, AS: Aserrín, SF: Suelo forestal). Las barras verticales indican el error estándar y las letras diferentes las diferencias significativas entre niveles dentro del factor (Prueba de Tukey, $\mathrm{P}<0,05 ; \mathrm{N}=3$ ).

Figura $\mathrm{N}^{\circ} 5$

RESPUESTA DE LAS PLANTAS SEGÚN LAS DIFERENTES VARIABLES EVALUADAS EN EL ENSAYO DE ESTABLECIMIENTO SEGÚN SUSTRATO Y CONTENEDOR

\section{Cuadro $\mathrm{N}^{\circ} 4$}

COSTO DE PRODUCCIÓN SEGÚN VALOR DE SUSTRATO, FERTILIZANTE, CONTENEDOR, TRANSPORTE, VOLUMEN DE SUSTRATO Y CAPACIDAD DE PLANYAS POR CONTENEDOR

\begin{tabular}{|l|c|c|c|}
\hline \multicolumn{2}{|c|}{ Tratamiento } & \multirow{2}{*}{ Valor Relativo } & (\%) \\
\hline \multirow{4}{*}{ Sustrato } & Contenedor & & 0,0 \\
\hline \multirow{4}{*}{ Aserrín } & Pequeño & 1,00 & 33,2 \\
\cline { 2 - 4 } & Mediano & 1,33 & 84,1 \\
\cline { 2 - 4 } & Grande & 1,84 & 4,9 \\
\hline \multirow{4}{*}{ Turba perlita forestal } & Pequeño & 1,05 & 39,8 \\
\cline { 2 - 4 } & Mediano & 1,40 & 93,9 \\
\cline { 2 - 4 } & Grande & 1,94 & 43,5 \\
\hline & Pequeño & 1,43 & 92,7 \\
\cline { 2 - 4 } & Mediano & 1,93 & 172,0 \\
\cline { 2 - 4 } & Grande & 2,72 & \\
\hline
\end{tabular}



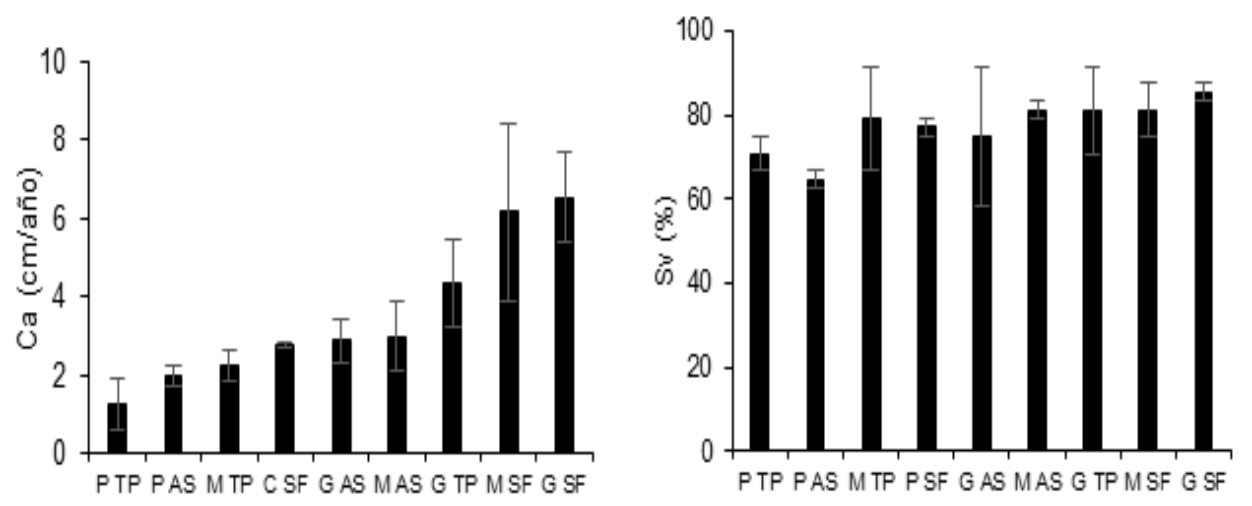

Crecimiento en altura (Ca) y sobrevivencia (Sv) con relación al tamaño del contenedor (P: Pequeño, M: Mediano, G: Grande) y el tipo de sustrato (Tp: Turba perlita, As: Aserrín, Sf: Suelo forestal).

Las barras verticales indican el error estándar y las letras diferentes las diferencias significativas entre tratamientos (Prueba de Tukey, $\mathrm{p}<0,05 ; \mathrm{n}=2$ ).

Figura $\mathrm{N}^{\circ} 6$

RESPUESTA DE LAS PLANTAS SEGÚN VARIABLES EVALUADAS EN EL ENSAYO DE ESTABLECIMIENTO SEGÚN CONTENEDOR Y SUSTRATO

\section{CONCLUSIONES}

El tipo de semilla asociado al color del cono no afectó el desempeño de pino ponderosa.

En el vivero y el sitio de plantación, las plantas con suelo forestal y contenedor de mayor volumen y profundidad presentaron el mayor tamaño, peso y crecimiento, y en general, valor de desempeño de las plantas.

La combinación más adecuada para desarrollar pino ponderosa fue el contenedor mediano con suelo forestal.

Este sustrato exhibió ventajas con relación al aserrín, debido al mejor desempeño de las plantas en todos los tamaños de envase a un costo equivalente.

El suelo forestal aventajó a la turba perlita debido al costo económico y ambiental sustancialmente menores.

Las plantas en contenedor mediano presentaron un crecimiento y calidad semejantes a las del grande, pero a un costo económico significativamente menor.

Los ensayos futuros deberían determinar la dosis óptima de fertilizante para cada sustrato y el efecto sobre el costo de producción.

\section{RECONOCIMIENTOS}

J. Chuquer.

Se agradece la colaboración prestada por V. Mondino, T. Schinelli, C. Buduba, P. Rago y 


\section{REFERENCIAS}

Bárbaro, L. A.; Imhoff, S. y Morisigue, D. E., 2014. Evaluación de sustratos formulados con corteza de pino, pinocha y turba subtropical. Ciencia del suelo 32(2): 149-158.

Bernaola-Paucar, R. M.; Barrios, E. P.; González, P. G.; Chaparro, V. M.; Santiago, G. A. y Pérez, E. S., 2015. Efecto del volumen del contenedor en calidad y supervivencia de Pinus hartwegii Lindl. en sistema dobletrasplante. Revista Mexicana de Ciencias Forestales 6(28): 174-187.

Buamscha, M. G.; Contardi, L.; Dumroese, R. K.; Enricci, J.; Escobar, R.; Gonda, H.; Jacobs, D .F.; Landis, T. D.; Luna, T.; Mexal, J. G. y Wilkinson, K., 2012. Producción de plantas en viveros forestales. Universidad Nacional de la Patagonia San Juan Bosco. Argentina: CFI - CIEFAP - UPSJB. 195 p.

Callaham, R. Z., 2013. Pinus ponderosa: geographic races and subspecies based on morphological variation. USA: USDA Forest Service Research Paper PSW-RP-265. 54 p.

Close, D. C.; Paterson, S.; Corkrey, R. and Mc Arthur, C., 2010. Influences of seedling size, container type and mammal browsing on the establishment of Eucalyptus globulus in plantation forestry. New Forests 39(1): 105-115.

Dickson, A.; Leaf, A. L. and Hosner, I. E., 1960. Quality appraisal of White Spruce and White Pine seedlings stock in nurseries. The Forestry Chronicle 36: 10-13.

Dobner, M.; Trazzi, P. A., Higa, A. e Seitz, R., 2013. Influência do volume do tubete e do método de plantio no crescimento de um povoamento de Pinus taeda aos nove anos de idade. Scientia Forestalis 41(97): 7-14.

Domínguez-Lerena, S.; Sierra, N. H.; Manzano, I. C.; Bueno, L. O.; Rubira, J. L. and Mexal, J. C., 2006. Container characteristics influence Pinus pinea seedling development in the nursery and field. Forest Ecology and Management 221(1-3): 63-71.

Gallo, L.; Martínez Meier, A.; Azpilicueta, M. M.; Marchelli, P. y Mondino, V., 2005. Subprograma coníferas y otras especies en la región patagónica. En: C.A Norverto (Ed). Mejores árboles para más forestadores: el programa de producción de material de propagación mejorado y el mejoramiento genético en el Proyecto Forestal de Desarrollo (p. 95-116). Argentina: Secretaría de Agricultura, Pesca y Alimentos.

Gerding, V.; Hermosilla, M. E. y Grez, R., 1996. Sustratos de corteza compostada para la propagación vegetativa de estacas de tallo de Podocarpus nubigena Lindl. y Eucryphia cordifolia Cav. Bosque 17(2): 57-64.

Gonda, H.; Davel, M.; Bonino, N.; Contardi, L.; Focarazzo, S.; Calvi, F.; Rush, V. y Mele, U., 2015. El establecimiento de las plantas. En: L. Chauchard, C. Frugoni, y C. Nowak (Eds.). Buenas prácticas para el manejo de plantaciones forestales en el noroeste de la Patagonia (p. 166-190). Argentina: Ministerio de Agroindustria.

Hartmann, H. T.; Kester, D. E.; Davies F.T. and Geneve, R. L., 2010. Hartmann y Kester's plant propagation: principles and practices. USA: Pearson. $1024 \mathrm{p}$.

INTA, 2017. http://inta.gob.ar/ documentos/datos-meteorologicos-de-el-maiten. Instituto Nacional de Tecnología Agropecuaria (INTA). Consultado 20-03-2017.

Iverson, R. D., 1984. Planting stock selection: Meeting biological needs and operational realities. En: M.L. Duryea, y T.D. Landis (Eds.). Forest nursery manual: production of bareroot seedlings (p. 261-266). The Netherlands: Martinus Nijhoff/Dr W. Junk Publishers.

Jones, B., 2001. Laboratory guide for conducting soil tests and plant analysis. USA: CRC Press.

Landis, T. D. and Dumroese, R. K., 2006. Applying the Target Plant Concept to Nursery Stock Quality. En: L. MacLennan, y J. Fennessy (Eds.). Plant quality: A key to success in forest establishment (p. 1-10). Ireland: Proceedings of the COFORD Conference, National Council for Forest Research and Development.

Landis, T.D. y Morgan, N., 2009. Growing media alternatives for forest and native plant nurseries. En: R. K. Dumroese, y L. E. Riley (Eds.). National Proceedings: Forest and Conservation Nursery Associations (p. 26-31). USA: Department of Agriculture, Forest Service, Rocky Mountain Research Station.

Larcher, F. and Scariot, V., 2009. Assessment of partial peat substitutes for the production of Camellia japonica. Hortscience 44(2): 312-316.

MAGyP, 2014. Argentina: Plantaciones forestales y gestión sostenible. Argentina: Ministerio de Agricultura, Ganadería y Pesca de la Nación. 
McTague, J. P. and Tinus, R. W., 1996. The effects of seedling quality and forest site weather on field survival of Ponderosa Pine. Tree Planters' Notes 47(1): 16-23.

Olivo, V. B. y Buduba, C. G., 2006. Influencia de seis sustratos en el crecimiento de Pinus ponderosa producido en contenedores bajo condiciones de invernáculo. Bosque 27(3): 267-271.

Oyarzábal, M.; Clavijo, J.; Oakley, L.; Biganzoli, F.; Tognetti, P.; Barberis, I.; Maturo, H. M.; Aragón, R.; Campanello, P. I.; Prado, D.; Oesterheld, M. y León, R., 2018. Unidades de vegetación de la Argentina. Ecología Austral 28: 40-63.

Paruelo, J.; Beltrán, A.; Jobbágy, E.; Sala, O. and Golluscio, R., 1998. The climate in Patagonia: General patterns and controls on biotic processes. Ecología Austral 8: 85-101.

Pinto, J. R.; Marshall, J. D.; Dumroese, R. K.; Davis, A. S. and Cobos, D. R., 2011. Establishment and growth of container seedlings for reforestation: A function of stocktype and edaphic conditions. Forest Ecology and Management 261(11): 1876-1884.

Quiroz, I.; Pincheira, M. P.; Hernández, J. A.; González, M.;García, E. y Soto, H., 2014. Efecto del volumen radicular sobre el crecimiento de Acacia dealbata Link. en vivero y en terreno en el secano de la región del Bio Bio, Chile. Revista Árvore 38(1): 155-164.

SAMLA, 1996. Sistema de apoyo metodológico para laboratorio de análisis de suelos y aguas. Argentina: Secretaría de Agricultura, Ganadería, Pesca, Alimentos y Forestación.

Schmidt-Vogt, H., 1981. Morphological and physiological characteristics of planting stock: present state of research and research tasks for the future ( $p$ 433-446). En: Proceedings IUFRO - XVII World Congress. Japan.

South, D. B.; Harris, S. W.; Barnett, J. P.; Hainds, M. J. and Gjerstad, D. H., 2005. Effect of container type and seedling size on survival and early height growth of Pinus palustris seedlings in Alabama, USA. Forest Ecology and Management 204(2-3): 385-398.

Varela, R., 2014. Manual de geología. Argentina: Instituto Superior de Correlación Geológica. 158 p. 
